\title{
Os periódicos de maior impacto na pesquisa contábil brasileira e norte- americana: Uma análise comparativa baseada nas citações em teses de doutorado
}

Octavio Ribeiro de Mendonça Neto

Doutor em Contabilidade (FEA
IUSP).Coordenador do Núcleo de Pesquisas
em Controladoria e Professor do Programa
de Pós-Graduação em Ciências Contábeis
do Centro de Ciências Sociais Aplicadas da
Universidade Presbiteriana Mackenzie
(CCSA)

Ricardo Lopes Cardoso

Doutor em Contabilidade pela FEA/USLP. Professor do Programa de Pós-Graduação em Ciências Contábeis do Centro de Ciências Sociais e Aplicadas da Universidade Presbiteriana Mackenzie (CCSA)

José Carlos Tiomatsu Oyadomari

Doutor em Contabilidade pela FEA/USLP. Professor do Programa de PósGraduação em Ciências Contábeis do Centro de Ciências Sociais e Aplicadas da Universidade Presbiteriana Mackenzie (CCSA)

O objetivo deste artigo é construir um ranking, baseado nas citações em teses de doutorado dos periódicos de língua inglesa com maior influência sobre a formação do pensamento contábil no Brasil. O estudo se caracteriza como descritivo quanto aos objetivos, documental (archive) quanto aos procedimentos e quantitativo em relação à abordagem do problema. Para construir esse ranking e com o intuito de facilitar a análise comparativa dos resultados obtidos com os de Chan et al. (2009), que realizaram o mesmo tipo de estudo para o caso norteamericano, utilizou-se a mesma metodologia proposta por esses autores, que é baseada na análise de citações. A base de dados utilizada é constituída pelas teses de doutorado da Faculdade de Economia, Administração e 
Contabilidade (FEA) da Universidade de São Paulo, defendidas no Programa de Pós-Graduação em Ciências Contábeis do Departamento de Contabilidade e Atuária, entre 11 de outubro de 1996 e 29 de julho de 2009. Os resultados obtidos estão em linha com aqueles de Chan et al. (2009) e, da mesma maneira que esses, fornecem evidências de que os periódicos mais citados variam de acordo com a área específica da contabilidade e o método de pesquisa utilizado pelo pesquisador. No caso do Brasil, os resultados revelam que os periódicos mais citados são: Journal of Accounting and Public Policy, The Accounting Review, The Journal of Finance, Accounting, Organizations and Society.

Palavras chave: Pesquisa contábil; Ranking de periódicos; Análise de citações.

\section{The journals of higher impact on accounting research in Brazil and USA. A comparative analysis based on doctoral theses citations}

The main purpose of this paper is the construction of a ranking of the English language journals that most influences formation of the accounting thought in Brazil, based on citations in doctoral theses. The paper has a descriptive characteristic; an archival procedure and quantitative approach has been adopted. In the construction of this journal ranking, we have used the methodology of Chan and others (2009) based on citations analysis to facilitate a comparative analysis with their study which was the American case. The data base used was the doctoral theses on accounting from the Faculty of Economy, Business and Accounting of São Paulo University defended between 1996 and 2009. The results are in accordance with those of Chan and others (2009), and also provide evidence that rankings of top journals vary by specialty area and research method. In the Brazilian case, the top journals cited are Journal of Accounting and Public Policy, The Accounting Review, The Journal of Finance, Accounting, Organizations and Society. 
Keywords: Accounting research; Journal ranking; Citation analysis.

Recebido em 01.05.2010 Aceito em 30.07.2011

\section{Introdução}

A grande maioria dos artigos publicados em periódicos científicos contém citações que indicam os estudos precedentes, ilustram e dão suporte ao que o autor tem para dizer. São ligações formais e explícitas entre estudos que têm pontos em comum (GARFIELD, 1979). Dessa forma, os pesquisadores, ao desenvolverem seus estudos, normalmente procuram fundamentá-los em trabalhos publicados em periódicos que percebam como de alta qualidade, sendo que essa percepção é, normalmente, fundamentada no ranking atribuído aos periódicos pela comunidade acadêmica as quais pertencem.

Conforme observam Ballas e Theoharakis (2003), uma visão idealística da ciência pode sugerir que a avaliação dos achados de uma pesquisa seja independente e igualmente aceita em todo o mundo. Todavia, a descoberta de variações significativas na percepção da qualidade dos periódicos entre a comunidade acadêmica, indica que se devem considerar fatores contextuais, antes de se classificar periódicos e avaliar os resultados de uma pesquisa acadêmica. No estudo realizado por esses autores, os resultados obtidos sugerem uma variação significativa na percepção da qualidade dos periódicos conforme a origem geográfica dos pesquisadores, linha de pesquisa, etc. Esses resultados confirmam os encontrados por Lukka e Kasanen (1996), que investigaram o grau de interação entre acadêmicos trabalhando em diferentes países e concluíram que a pesquisa acadêmica ainda é localmente focada.

Mais recentemente, Chan et al. (2009), com o objetivo de propor uma nova metodologia de indexação para periódicos científicos em contabilidade, analisaram as citações em teses de doutorado da área, disponíveis na base de dados PROQUEST Dissertations and Theses, durante o período de janeiro de 1999 a dezembro de 2003. Neste estudo, onde classificaram essas teses e dissertações conforme as áreas específicas da contabilidade (auditoria, contabilidade financeira, contabilidade gerencial, sistemas de informação e contabilidade tributária) e conforme os métodos de pesquisa adotados (archival, experimental, modelagem e survey), os autores concluíram que a qualidade dos periódicos, avaliada em função do número de citações, varia conforme a área da contabilidade em que a pesquisa foi realizada e o método utilizado.

Conforme observam Chan et al. (2009), as teses de doutorado constituem o principal trabalho desenvolvido pelos novos doutores. Esse 
trabalho é supervisionado por professores doutores, examinado e sancionado por uma banca, constituída por acadêmicos experientes, inclusive de outras universidades, representando, desta forma, uma pesquisa profunda, realizada por novos pesquisadores, em um ambiente de rigor acadêmico. Citações em teses de contabilidade apresentam a fundamentação das pesquisas desses novos acadêmicos, revelando, assim, a influência dos periódicos em seu trabalho.

Dessa forma, o presente estudo tem por objetivo apresentar um ranking dos periódicos de língua inglesa que têm maior influência sobre a formação do pensamento contábil no Brasil, baseado, da mesma forma que Chan et al. (2009), nas citações em teses de doutorado. Esse estudo também compara os resultados obtidos por esses autores, fornecendo, assim, uma visão do grau de alinhamento entre a pesquisa contábil brasileira e americana. Pretende-se, ainda, que esses resultados possam contribuir para o aperfeiçoamento do processo de indexação de periódicos adotado pela Coordenação de Aperfeiçoamento de Pessoal de Nível Superior (CAPES) e pela comunidade acadêmica brasileira, de uma forma geral.

Na seção 2, apresenta-se um panorama da literatura sobre o assunto, enquanto a seção 3 discute a metodologia utilizada e a seção 4 analisa os resultados obtidos, além de compará-los com os de Chan et al. (2009). As considerações finais, contribuições e limitações são apresentadas na seção 5 .

\section{Revisão da literatura}

Conforme Garfield (1979), o conceito de indexação de citações é bastante simples. A grande maioria dos documentos publicados em um periódico científico, sejam eles artigos, resenhas, relatórios de pesquisa, etc., contém citações. Essas citações, identificadas pelo nome do autor, título, veículo e data em que foram publicadas, fornecem informações sobre documentos precedentes que fundamentam ou ilustram o que o autor da citação pretende dizer. Dessa forma, as citações constituem uma ligação formal e explícita entre documentos que têm pontos em comum. Assim, qualquer pesquisador pode encontrar vários documentos adicionais sobre um determinado assunto, consultando apenas um documento citado e qualquer consulta a esses documentos adicionais fornece uma nova lista de citações, o que possibilita a continuidade da pesquisa.

Ainda de acordo com Garfield (1979), a análise de citações é utilizada para avaliar os periódicos científicos, bem como os pesquisadores que neles publicam. As ligações das citações entre artigos científicos fornecem uma medida relevante da utilidade de um determinado periódico científico. Todavia, a visão proporcionada pelas citações não é definitiva, já que o mérito científico não é a única razão para que um autor cite um 
artigo publicado. Alguns fatores, como a reputação do autor citado e a visibilidade, prestígio e acessibilidade do periódico em questão, podem influenciar na escolha do artigo a ser citado. Em função disso, a visão proporcionada pela quantidade de citações de periódicos deve ser utilizada com cuidado, aplicada somente quando for relevante e, além disso, deve ser interpretada dentro do contexto de uma decisão a ser tomada ou de uma hipótese a ser testada. Apesar dessa limitação, continua o autor (GARFIELD, 1979), dados sobre citações em periódicos contém muitas informações úteis. Conhecer a taxa de citações de um periódico, as fontes das referências que o citam e os periódicos que ele cita, permite mensurar sua utilidade como fonte de informação para pesquisa, caracteriza sua linha editorial e, dependendo do periódico, define a literatura fundamental de uma especialidade ou disciplina.

Existem, na literatura, vários estudos focados na avaliação da qualidade dos periódicos. Dentre esses, Bonner et al. (2006) apresentam uma revisão dos principais trabalhos publicados sobre o tema até 2004. Neste artigo, que resume os achados de 16 pesquisas, os autores observam que cinco periódicos - Accounting, Organizations and Society (AOS), Contemporary Accounting Research (CAR), Journal of Accounting and Economics (JAE), Journal of Accounting Research (JAR) e The Accounting Journal (TAR) - aparecem constantemente como os mais importantes em contabilidade. Salientam, ainda, que, nos estudos focados em áreas específicas da contabilidade, encontram-se algumas variações na ordem de importância dos mesmos. Enquanto que JAE, JAR e TAR são normalmente ranqueados acima de $A O S$ e $C A R$ em contabilidade financeira, AOS, JAR e TAR aparecem acima de CAR e JAE, no campo da contabilidade gerencial.

Desses 16 estudos, 13 baseiam-se na percepção de acadêmicos e profissionais, três na análise de citações e um mensura a qualidade dos periódicos com base no número de downloads da base de dados Social Science Research Network (SSRN), durante o período 1999 - 2001.

Já Chan et al. (2009) apresentam, em seu estudo, uma revisão dos trabalhos não incluídos ou publicados posteriormente ao estudo realizado por Bonner et al. (2006). Essa revisão compreende os estudos de Brown (2003), Heron e Hall (2004), Reinstein e Calderon (2006), Lowenson e Samuelson (2006), Beattie e Goodacre (2006) e Chan e Liano (2009 apud CHAN et al., 2009), dos quais, quatro baseiam-se na percepção de acadêmicos e profissionais, um na análise de citações e um no número de downloads da base de dados Social Science Research Network (SSRN). Os resultados obtidos são semelhantes àqueles dos trabalhos revistos por Bonner et al. (2006), embora incluam alguns periódicos não mencionados anteriormente entre os cinco mais citados, como o National Tax Journal (NAT), Journal of American Taxation Association (JATA), Accounting Historians Journal (AHJ), Journal of Business Finance and Accounting 
(JBFA) e Behavioral Research in Accounting (BRIA), o que sugere uma percepção de qualidade diferenciada, em função da linha de pesquisa dos respondentes.

O trabalho de Chan et al. (2009) é baseado em citações de teses de doutorado e complementa os estudos revisados por eles e por Bonner et al. (2006), construindo tanto um ranking geral como rankings específicos, de acordo com as áreas da contabilidade e com o método de pesquisa utilizado. Para construção desses rankings, os autores analisaram os periódicos citados em 247 teses de doutorado disponíveis na base de dados PROQUEST Dissertations and Theses, referentes ao período de janeiro de 1999 a dezembro de 2003. Essas 247 teses representam 84 universidades americanas e 6 de outros países de língua inglesa, os quais contribuíram com apenas 11 teses. Os resultados obtidos revelam rankings diferentes, tanto na dimensão relacionada com as áreas específicas da contabilidade como na dimensão relacionada ao método de pesquisa. Estes resultados são apresentados em detalhes, na seção 4 do presente estudo.

Por último, além dos trabalhos revisados por Bonner et al. (2006) e, ainda, Chan et al. (2009), merecem destaque duas pesquisas relacionadas ao tema. A primeira delas, realizada por Bean e Bernardi (2005), na qual os autores desenvolveram um modelo para estimar a qualidade de periódicos. Este modelo foi construído utilizando uma regressão, que teve como variáveis independentes três características dos periódicos (idade, taxa de aceitação e público alvo) e como variável dependente os scores de percepção, levantados anteriormente por Ballas e Theoharakis (2003). As hipóteses levantadas pelos autores são as de que: a) quanto maior for a idade de um periódico, maior será sua qualidade; b) quanto maior for a taxa de aceitação de um periódico, menor será sua qualidade; e c) os periódicos destinados ao público acadêmico têm mais qualidade do que aqueles destinados aos profissionais. O modelo em questão foi testado com os resultados de nove estudos diferentes, baseados em percepção, e apresentou um $R^{2}$ ajustado médio de 35,9\%.

A segunda pesquisa que cabe ainda ser mencionada é a de Matherly e Shortridge (2009). Neste estudo os autores criticam os dois principais métodos utilizados para mensurar a qualidade dos periódicos, a saber: a análise de citações e a percepção de acadêmicos e propõem um novo modelo de análise. Segundo eles a análise de citações, que assume que o número de citações recebidas por um periódico é uma proxie de sua qualidade, apresenta vários problemas, dentre eles: o fato de que alguns periódicos e autores possam ser citados apenas por sua reputação e de que muitos artigos são citados apenas com o objetivo de serem refutados. Com relação à percepção dos acadêmicos, observam que o método parte do princípio que os acadêmicos consultados estão familiarizados com uma 
larga gama publicações o que, dado o elevado e crescente número de periódicos, torna-se, cada vez, menos provável.

Matherly e Shortridge (2009) concordam com as premissas do modelo desenvolvido por Bean e Bernardi (2005) e constroem um novo modelo, com o objetivo de aprimorá-lo e obter resultados estatísticos mais significativos. No modelo desenvolvido por Matherly e Shortridge (2009), a variável dependente corresponde a uma média dos resultados da percepção de acadêmicos obtidos em cinco estudos precedentes, dois mais recentes de Johnson, Reckers e Solomon (2002) e Herron e Hall (2004), e três mais antigos, Brown e Huefner (1994), Smith (1994) e Jolly, Schroeder e Spear (1995). Desta forma, os autores incluem em seu modelo as opiniões de diversos grupos de acadêmicos ao longo do tempo. Como variáveis independentes consideraram as seguintes: 1) o público alvo do periódico (acadêmicos ou profissionais); 2) a extensão média dos artigos; 3) acessibilidade do periódico (avaliada conforme sua disponibilidade nas bases de dados $A B I-$ Inform, EBSCO e H. W. Wilson); 4) inclusão no Social Sciences Citation Index; e 5) custo de submissão de artigo ao periódico. Como as variáveis independentes 1 e 2 apresentaram alto grau de correlação $(r=0,64)$, os autores só utilizaram no modelo a variável 2, ou seja, a extensão média dos artigos.

Os resultados obtidos sugerem que os cinco periódicos de contabilidade, percebidos como de maior qualidade são, em ordem decrescente: Journal of Accounting Research (JAR); Journal of Accounting and Economics (JAE); Contemporary Accounting Research (CAR); The Accounting Review (TAR) e Review of Accounting Studies (RAS). Segundo os autores, o score obtido pelo Accounting, Organizations and Society (110 lugar), ficou abaixo do esperado e uma possível explicação para isso, seria a de que o periódico não cobra pela submissão de artigos. Isso, segundo os autores, ilustra que o julgamento subjetivo continua sendo necessário ao se aplicar um modelo quantitativo em decisões subjetivas.

\section{Metodologia}

O presente estudo se caracteriza como de natureza descritiva quanto a seus objetivos, do tipo documental (archival) quanto a seus procedimentos e quantitativo, em relação à abordagem do problema. Para construir um ranking de periódicos, utilizou-se a mesma metodologia proposta por Chan et al. (2009), baseada na análise de citações. A escolha da mesma metodologia, sem nenhuma adaptação, foi deliberada e teve por objetivo facilitar a análise comparativa dos resultados.

Essa metodologia consistiu em analisar todas as 55 teses de doutorado disponíveis na base de dados Teses e Dissertações da Faculdade de Economia, Administração e Contabilidade (FEA) da Universidade de São Paulo, defendidas no Programa de Pós-Graduação em Ciências Contábeis do Departamento de Contabilidade e Atuária. Cabe 
ressaltar que até recentemente, este era o único programa, no Brasil, que oferecia um curso de Doutorado em Contabilidade e que os outros cursos recentemente autorizados a funcionar, até a presente data, não tiveram nenhum processo de defesa de teses concluído. Isso posto, foram examinadas as citações de periódicos internacionais em 55 teses de doutorado, defendidas no horizonte de tempo que vai de 11 de outubro de 1996 a 29 de julho de 2009.

Esta análise foi realizada obedecendo às seguintes etapas:

Etapa 1: Levantamento de todas as 55 teses de doutorado disponíveis na base de dados já mencionadas;

Etapa 2: Classificação dessas teses por áreas específicas da contabilidade e por método de pesquisa utilizado, com o objetivo de identificar os periódicos internacionais mais citados, de acordo com essas duas dimensões de análise. Da mesma forma que o fizeram Chan et al. (2009), as áreas específicas utilizadas foram auditoria (auditing), financeira (financial), gerencial (managerial), sistemas (systems) e tributária (tax). As teses que não se enquadraram nessas cinco áreas específicas foram classificadas como "outro". Os métodos de pesquisa foram classificados em documental (archival), experimental (experimental), modelagem (modeling) e levantamento (survey). Novamente, as teses que não se enquadraram nesses quatro métodos de pesquisa foram classificadas como "outro". Ainda, seguindo a metodologia proposta por Chan, Chan, Seow e Tam (2009), as classificações foram efetuadas por dois pesquisadores separadamente $e$, quando não houve concordância, o terceiro pesquisador apresentou seu julgamento;

Etapa 3: Levantamento dos periódicos internacionais citados nessas teses e registrados nas suas respectivas referências bibliográficas;

Etapa 4: Contagem do número de vezes em que um periódico aparece nas referências bibliográficas, realizada separadamente por área específica da contabilidade e método de pesquisa. Essa contagem foi realizada com a utilização dos recursos do software Microsoft Office Excel 2007; e

Etapa 5: Os resultados dessa contagem foram tratados com 0 auxílio de técnicas de estatística descritiva, comumente utilizadas neste tipo de estudo, ou seja: a) cálculo a frequência do número de teses por área específica e método de pesquisa; b) cálculo da frequência do número de citações por área específica e método de pesquisa; e c) cálculo do número de citações de cada periódico por área específica e método de pesquisa, ponderado pelo número de teses classificadas em cada uma das áreas específicas e métodos de pesquisa. Essa ponderação teve por objetivo mitigar os possíveis vieses introduzidos pela concentração de teses em uma determinada área específica ou método de pesquisa.

Os resultados obtidos são apresentados e analisados na sequência. 


\section{Resultados}

\subsection{Horizonte temporal}

As 55 teses analisadas foram defendidas no período de 11 de outubro de 1996 a 29 de julho de 2009 e estão distribuídas neste horizonte temporal, conforme a TAB. 1 .

Tabela 1 - Número de teses defendidas por ano

\begin{tabular}{cccccccccccccc}
\hline Ano & 1996 & 1998 & 2000 & 2001 & 2002 & 2003 & 2004 & 2005 & 2006 & 2007 & 2008 & 2009 & Total \\
$\mathrm{N}^{\circ}$ & 01 & 01 & 01 & 02 & 02 & 03 & 03 & 08 & 08 & 09 & 11 & 06 & 55 \\
\hline
\end{tabular}

Fonte: Dados da pesquisa.

Esses dados mostram que as defesas dessas teses estão concentradas a partir de 2004 e representam $76 \%$ do universo analisado (42 em um total de 55).

\subsection{Frequência descritiva}

A seguir, na TAB. 2, são apresentadas as frequências das teses analisadas (Painel A) e de suas respectivas citações de periódicos de língua inglesa (Painel B), classificadas, conforme as duas dimensões propostas, ou seja, por área específica e por método de pesquisa. Como na análise realizada não se encontrou nenhuma tese na área de sistemas, essa área específica não foi considerada.

Tabela 2 - Número de teses e citações de periódicos por área específica e método de pesquisa

\begin{tabular}{|c|c|c|c|c|c|}
\hline Método de & Áreas de Especialidade & & & & \\
\hline Pesquisa & Financeira & Gerencial & Tributária & Outras & Total \\
\hline
\end{tabular}

Painel A: Número de teses por área de especialização e método de pesquisa

$\begin{array}{lllllll}\text { Documental } & 1 & 23 & 2 & 5 & 1 & 32 \\ \text { Experimental } & & & & & 1 & 1 \\ \text { Modelagem } & 1 & 1 & & 1 & & 3 \\ \text { Levantamento } & & 5 & 9 & & 3 & 17 \\ \text { Outros } & 2 & 29 & 13 & 6 & 5 & 2 \\ \text { Total } & 2 & & & & & 55\end{array}$

Painel B: Número de citações por área de especialização e método de pesquisa

$\begin{array}{lllllll}\text { Documental } & 5 & 1017 & 31 & 281 & 12 & 1346 \\ \text { Experimental } & & & & 3 & 3\end{array}$


Os periódicos de maior impacto na pesquisa contábil brasileira e norte-americana: uma análise comparativa baseada nas citações em teses de doutorado
Octavio Ribeiro de Mendonça Neto; Ricardo Lopes Cardoso; José Carlos Tiomatsu Oyadomari
Modelagem 3
78
8
89
Levantamento
155
472
161
788
Outros
84
84
Total
8
1250
587
289
176
2310

Fonte: Dados da pesquisa.

Os dados do Painel $A$ revelam uma predominância de teses na área de contabilidade financeira ( 29 em 55, ou seja, 53\%), seguindo-se a área de contabilidade gerencial (13 em 55, ou seja, 24\%). Esses resultados estão em linha com os levantados por Chan et al. (2009), cujos percentuais obtidos foram de $55 \%$ e $15 \%$, respectivamente. Com relação aos métodos de pesquisa utilizados, observa-se que na área de contabilidade financeira, a pesquisa documental foi a mais frequente (79\%), enquanto que na área da contabilidade gerencial predominou o levantamento (69\%). No caso da pesquisa de Chan et al. (2009), a do tipo documental também é predominante na área de contabilidade financeira $(93 \%)$, mas, no caso da contabilidade gerencial, os resultados apresentam uma diferença importante, uma vez que o levantamento só foi utilizado em $27 \%$ das teses, predominando a pesquisa experimental $(51 \%)$.

Já no Painel $B$, observa-se que as pesquisas em contabilidade financeira, que utilizaram a pesquisa documental, foram responsáveis pela maior parte das citações, 1017 em 1250 (81\%), resultado este condizente com os do Painel A e, também, em linha com os $93 \%$ obtidos por Chan, Chan, Seow e Tam (2009). Com relação à contabilidade gerencial, como seria de se esperar, tendo em conta os dados do Painel $A$, os resultados obtidos diferem dos de Chan, Chan, Seow e Tam (2009) (44\% das citações estão relacionadas com os experimentos) e mostram que as pesquisas que utilizaram o levantamento foram as responsáveis pela maioria das citações na área $(80 \%)$.

\subsection{Citações por periódicos e por método de pesquisa utilizado, ponderado pelo número de teses}

Da mesma forma que Chan et al. (2009), a TAB 3 apresenta um ranking dos periódicos mais citados, de acordo com o método de pesquisa utilizado.

Para facilitar a leitura dos dados, o QUADRO. 1, a seguir, apresenta uma listagem das abreviaturas utilizadas para designar os periódicos citados.

Quadro 1 - Títulos abreviados dos periódicos

\begin{tabular}{cccc} 
Título & Título completo & Título & Título completo \\
Abrevi & & Abrevia & \\
\hline
\end{tabular}

Perspectivas em Ciência da Informação, v.16, n.3, p.93-115 jul./set. 2011 
ado do

AAA Accounting, Auditing and JFE Journal of Financial Economics
Accountability

AER American Accounting Review

AH Accounting Horizons

AMR Academic of Management Review

AOS

ASQ

CAR Contemporary Accounting Research

ECO Econometrica

FS Fiscal Studies

HBR Harvard Business Review

IAE Issues in Accounting Education

ITJ International Tax Journal

ITPF

JAL Journal of Accounting Literature

JAPP Journal of Accounting and Public Policy

JAR

Journal of Accounting Research

JATA Journal of American Taxation

JB Journal of Business

JBE Journal of Business Ethics

JBFA Journal of Business Finance and

Accounting
JFQA Journal of Financial Quantitative

Analysis

JIFMA Journal of Financial Management

Accounting

JLE Journal of Law and Economics

Journal of Law and Economics and

Organizations

JMAR Journal of Management Accounting

Research

JPE

MA

MS

NTJ

Journal of Political Economy

Management Accounting

Management Science

QJE

National Tax Journal

RAF

Quarterly Journal of Economics

SMJ

Review of Accounting and Finance

Southern Economic Journal

TAJS The American Journal of Sociology

TAMJ The Academy of Management Journal

TAR The Accounting Review

TJA The Journal of Accountancy

TJF The Journal of Finance

TJPE The Journal of Public Economics

Fonte: Dados da pesquisa.

Os dados apresentados na TAB. 3, consistem de 2310 citações, obtidas nas 55 teses analisadas, o que corresponde a uma média de 42 citações por tese. No estudo de Chan et al. (2009), que analisou 247 teses, essa média foi de 82 citações por tese. 
Ainda utilizando a metodologia dos referidos autores, o número de citações foi ponderado pelo número de teses, controlando-se, assim, um possível viés, introduzido pela predominância das pesquisas documentais.

Dessa forma, os resultados obtidos nas colunas 4, 6, 8, 10 e 12 foram somados para obter o resultado da coluna 14 , que caracteriza 0 ranking apresentado.

Com relação ao método de pesquisa, os estudos que utilizaram a metodologia da pesquisa documental foram responsáveis por $58,3 \%$ das citações, seguindo-se a metodologia do levantamento, com 34,1\%.

Tabela 3 - Citações por periódico e por método de pesquisa.

\begin{tabular}{|c|c|c|c|c|c|c|c|c|c|c|c|c|}
\hline \multirow{2}{*}{$\begin{array}{l}\text { Journal } \\
\text { (1) }\end{array}$} & \multirow{2}{*}{$\begin{array}{l}\text { Total } \\
\text { (2) }\end{array}$} & \multicolumn{2}{|c|}{$\begin{array}{l}\text { Documental } \\
32 \text { Teses }\end{array}$} & \multicolumn{2}{|c|}{$\begin{array}{l}\text { Levantamento } \\
17 \text { Teses }\end{array}$} & \multicolumn{2}{|c|}{$\begin{array}{l}\text { Experimental } \\
1 \text { Teses }\end{array}$} & \multicolumn{2}{|c|}{$\begin{array}{l}\text { Modelagem } \\
3 \text { Teses }\end{array}$} & \multicolumn{2}{|c|}{$\begin{array}{l}\text { Outros } \\
2 \text { Teses }\end{array}$} & \multirow{2}{*}{$\begin{array}{l}\text { Total } \\
\text { (13) }\end{array}$} \\
\hline & & $\begin{array}{l}\text { Cit. } \\
\text { (3) }\end{array}$ & $\begin{array}{l}\text { Cit./T } \\
\text { (4) }\end{array}$ & $\begin{array}{l}\text { Cit. } \\
(5)\end{array}$ & $\begin{array}{l}\text { Cit./T } \\
\text { (6) }\end{array}$ & $\begin{array}{l}\text { Cit. } \\
\text { (7) }\end{array}$ & $\begin{array}{l}\text { Cit./T } \\
(8)\end{array}$ & $\begin{array}{l}\text { Cit. } \\
(9)\end{array}$ & $\begin{array}{l}\text { Cit./T } \\
\text { (10) }\end{array}$ & $\begin{array}{l}\text { Cit. } \\
\text { (11) }\end{array}$ & $\begin{array}{l}\text { Cit./T } \\
\text { (12) }\end{array}$ & \\
\hline TJF & 107 & 78 & 2,44 & 5 & 0,29 & 0 & 0,00 & 23 & 7,67 & 1 & 0,50 & 10,90 \\
\hline JMAR & 64 & 2 & 0,06 & 46 & 2,71 & 0 & 0,00 & 0 & 0,00 & 16 & 8,00 & 10,77 \\
\hline AOS & 93 & 8 & 0,25 & 75 & 4,41 & 0 & 0,00 & 0 & 0,00 & 10 & 5,00 & 9,66 \\
\hline JAPP & 234 & 207 & 6,47 & 25 & 1,47 & 0 & 0,00 & 1 & 0,33 & 1 & 0,50 & 8,77 \\
\hline TAR & 171 & 126 & 3,94 & 40 & 2,35 & 1 & 1,00 & 4 & 1,33 & 0 & 0,00 & 8,62 \\
\hline AER & 46 & 25 & 0,78 & 9 & 0,53 & 1 & 1,00 & 10 & 3,33 & 1 & 0,50 & 6,14 \\
\hline JAR & 139 & 109 & 3,41 & 28 & 1,65 & 0 & 0,00 & 0 & 0,00 & 2 & 1,00 & 6,05 \\
\hline JIFMA & 25 & 10 & 0,31 & 2 & 0,12 & 0 & 0,00 & 13 & 4,33 & 0 & 0,00 & 4,76 \\
\hline JFQA & 16 & 6 & 0,19 & 0 & 0,00 & 0 & 0,00 & 10 & 3,33 & 0 & 0,00 & 3,52 \\
\hline AAA & 18 & 7 & 0,22 & 6 & 0,35 & 0 & 0,00 & 0 & 0,00 & 5 & 2,50 & 3,07 \\
\hline JFE & 76 & 71 & 2,22 & 3 & 0,18 & 0 & 0,00 & 2 & 0,67 & 0 & 0,00 & 3,06 \\
\hline JLEO & 7 & 0 & 0,00 & 1 & 0,06 & 0 & 0,00 & 0 & 0,00 & 6 & 3,00 & 3,06 \\
\hline JLE & 14 & 8 & 0,25 & 2 & 0,12 & 0 & 0,00 & 1 & 0,33 & 3 & 1,50 & 2,20 \\
\hline SMJ & 16 & 3 & 0,09 & 10 & 0,59 & 0 & 0,00 & 0 & 0,00 & 3 & 1,50 & 2,18 \\
\hline CAR & 47 & 34 & 1,06 & 12 & 0,71 & 0 & 0,00 & 1 & 0,33 & 0 & 0,00 & 2,10 \\
\hline $\mathrm{AH}$ & 56 & 51 & 1,59 & 5 & 0,29 & 0 & 0,00 & 0 & 0,00 & 0 & 0,00 & 1,89 \\
\hline JB & 13 & 8 & 0,25 & 1 & 0,06 & 0 & 0,00 & 4 & 1,33 & 0 & 0,00 & 1,64 \\
\hline JEP & 14 & 6 & 0,19 & 5 & 0,29 & 0 & 0,00 & 3 & 1,00 & 0 & 0,00 & 1,48 \\
\hline MS & 14 & 3 & 0,09 & 9 & 0,53 & 0 & 0,00 & 1 & 0,33 & 1 & 0,50 & 1,46 \\
\hline JBE & 26 & 4 & 0,13 & 22 & 1,29 & 0 & 0,00 & 0 & 0,00 & 0 & 0,00 & 1,42 \\
\hline JPE & 28 & 19 & 0,59 & 8 & 0,47 & 0 & 0,00 & 1 & 0,33 & 0 & 0,00 & 1,40 \\
\hline NTJ & 33 & 31 & 0,97 & 1 & 0,06 & 0 & 0,00 & 1 & 0,33 & 0 & 0,00 & 1,36 \\
\hline QJE & 22 & 10 & 0,31 & 11 & 0,65 & 0 & 0,00 & 1 & 0,33 & 0 & 0,00 & 1,29 \\
\hline TAMJ & 21 & 0 & 0,00 & 21 & 1,24 & 0 & 0,00 & 0 & 0,00 & 0 & 0,00 & 1,24 \\
\hline AMR & 5 & 1 & 0,03 & 2 & 0,12 & 0 & 0,00 & 0 & 0,00 & 2 & 1,00 & 1,15 \\
\hline RAF & 17 & 14 & 0,44 & 2 & 0,12 & 0 & 0,00 & 0 & 0,00 & 1 & 0,50 & 1,06 \\
\hline
\end{tabular}


Os periódicos de maior impacto na pesquisa contábil brasileira e norte-americana: uma análise comparativa baseada nas citações em teses de doutorado

Octavio Ribeiro de Mendonça Neto; Ricardo Lopes Cardoso; José Carlos Tiomatsu Oyadomari

\begin{tabular}{|c|c|c|c|c|c|c|c|c|c|c|c|c|}
\hline $\mathrm{ECO}$ & 16 & 12 & 0,38 & 3 & 0,18 & 0 & 0,00 & 0 & 0,00 & 1 & 0,50 & 1,05 \\
\hline ASQ & 9 & 0 & 0,00 & 8 & 0,47 & 0 & 0,00 & 0 & 0,00 & 1 & 0,50 & 0,97 \\
\hline TAJS & 9 & 1 & 0,03 & 7 & 0,41 & 0 & 0,00 & 0 & 0,00 & 1 & 0,50 & 0,94 \\
\hline JBFA & 17 & 14 & 0,44 & 2 & 0,12 & 0 & 0,00 & 1 & 0,33 & 0 & 0,00 & 0,89 \\
\hline Outros & 954 & 492 & & 419 & & 1 & & 13 & & 29 & & \\
\hline Total & 2310 & 1346 & & 788 & & 3 & & 89 & & 84 & & \\
\hline$\%$ & & 58,3 & & 34,1 & & 0,2 & & 3,8 & & 3,6 & & \\
\hline
\end{tabular}

Fonte: Dados da pesquisa.

Baseado nessa métrica, os cinco periódicos mais citados foram, pela ordem: The Journal of Finance (TJF), Journal of Managerial Accounting Research (JMAR), Accounting Organizations and Society (AOS), Journal of Accounting Policy e The Accounting Review (TAR).

Uma comparação entre esses resultados e aqueles obtidos por Chan et al. (2009) é apresentada no TAB. 4.

Tabela 4 - Análise comparativa das citações por periódico e por método de pesquisa

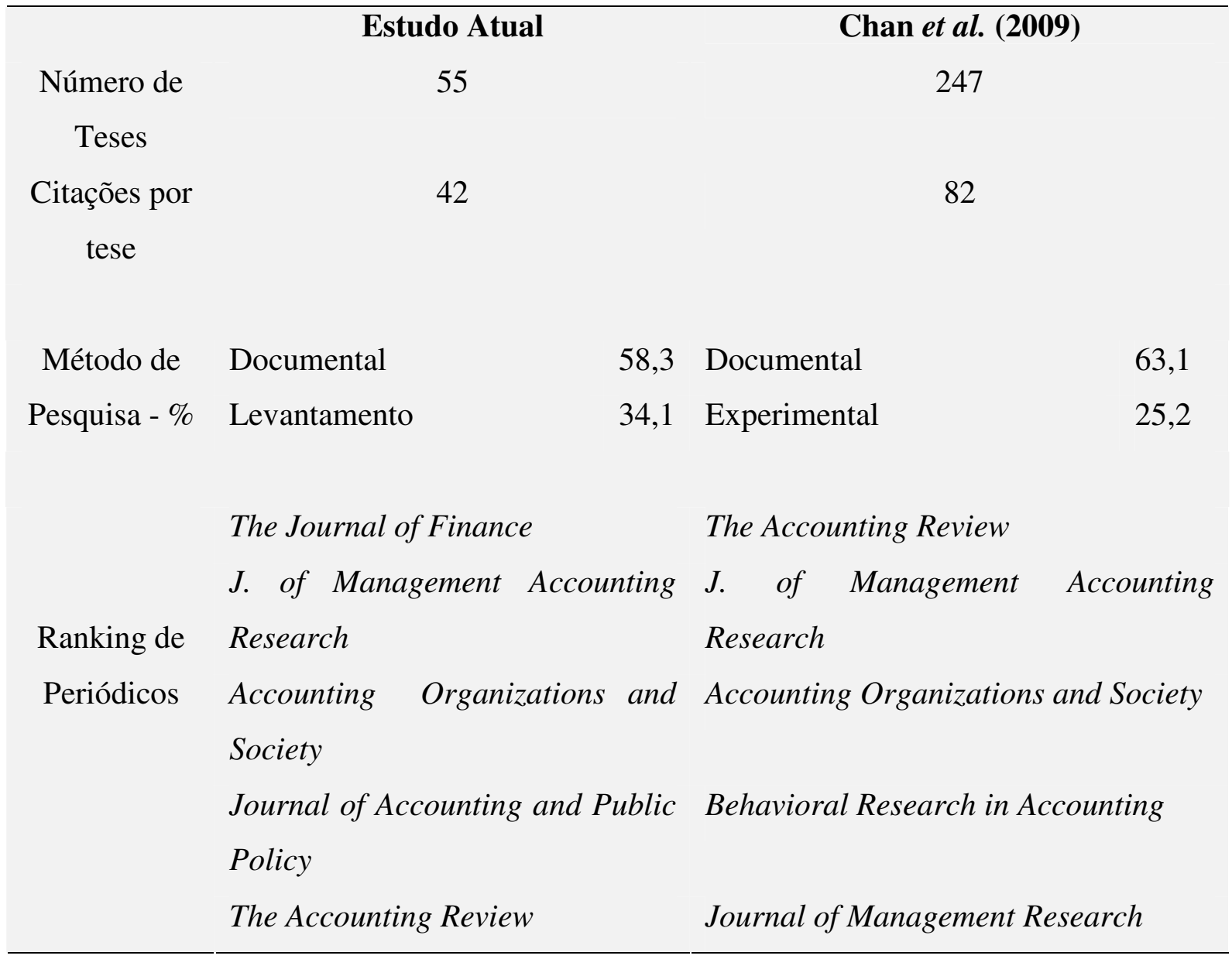

Fonte: Dados da pesquisa. 
Esses dados revelam que o método de pesquisa mais utilizado em ambos os casos é o documental, seguindo-se, no caso brasileiro, pelo levantamento e, no caso norte americano, pela pesquisa experimental. Com relação aos periódicos, observa-se uma coincidência de três deles, Journal of Management Accounting Research (JAMR), Accounting, Organizations and Society (AOS) e The Accounting Review (TAR), embora em ordem diferente nos respectivos rankings. No ranking de Chan, Chan, Seow e Tam (2009), o Behavioral Research in Accounting (BRIA) aparece na quarta posição, o que possivelmente está relacionado ao número relevante de teses que utilizam a metodologia experimental. Essa metodologia, que ainda é muito pouco explorada pelos pesquisadores brasileiros, requer um elevado grau de interdisciplinaridade com outras áreas do conhecimento, como a psicologia e a sociologia, o que apenas, recentemente, vem sendo incentivado no meio acadêmico contábil brasileiro.

\subsection{Citações de periódicos por área de especialização, ponderado pelo número de teses}

A TAB. 5, da mesma forma que Chan et al. (2009), apresenta um ranking dos periódicos mais citados, de acordo com a área de especialização. Essa tabela foi construída da mesma forma que a TAB. 3, apresentada no item 4.3.

Tabela 5 - Citações por periódico e por área de especialização.

\begin{tabular}{|c|c|c|c|c|c|c|c|c|c|c|c|c|}
\hline \multirow{2}{*}{$\begin{array}{l}\text { Journal } \\
\text { (1) }\end{array}$} & \multirow{2}{*}{$\begin{array}{l}\text { Total } \\
\text { (2) }\end{array}$} & \multicolumn{2}{|c|}{$\begin{array}{l}\text { Auditoria } \\
2 \text { Teses }\end{array}$} & \multicolumn{2}{|c|}{$\begin{array}{l}\text { Financeira } \\
29 \text { Teses }\end{array}$} & \multicolumn{2}{|c|}{$\begin{array}{l}\text { Gerencial } \\
13 \text { Teses }\end{array}$} & \multicolumn{2}{|c|}{$\begin{array}{l}\text { Tributária } \\
6 \text { Teses }\end{array}$} & \multicolumn{2}{|c|}{$\begin{array}{l}\text { Outras } \\
5 \text { Teses }\end{array}$} & \multirow{2}{*}{$\begin{array}{l}\text { Total } \\
\text { (13) }\end{array}$} \\
\hline & & $\begin{array}{l}\text { Cit. } \\
(3)\end{array}$ & $\begin{array}{l}\text { Cit./T } \\
\text { (4) }\end{array}$ & $\begin{array}{l}\text { Cit. } \\
(5)\end{array}$ & $\begin{array}{l}\text { Cit./T } \\
\text { (6) }\end{array}$ & $\begin{array}{l}\text { Cit. } \\
\text { (7) }\end{array}$ & $\begin{array}{l}\text { Cit./T } \\
(8)\end{array}$ & $\begin{array}{l}\text { Cit. } \\
(9)\end{array}$ & $\begin{array}{l}\text { Cit./T } \\
(10)\end{array}$ & $\begin{array}{l}\text { Cit. } \\
\text { (11) }\end{array}$ & $\begin{array}{l}\text { Cit./T } \\
\text { (12) }\end{array}$ & \\
\hline JAPP & 234 & 0 & 0,00 & 202 & 6,97 & 17 & 1,31 & 15 & 2,50 & 0 & 0,00 & 10,77 \\
\hline TAR & 171 & 0 & 0,00 & 122 & 4,21 & 30 & 2,31 & 16 & 2,67 & 3 & 0,60 & 9,78 \\
\hline AOS & 93 & 0 & 0,00 & 13 & 0,45 & 76 & 5,85 & 1 & 0,17 & 3 & 0,60 & 7,06 \\
\hline JAR & 139 & 0 & 0,00 & 117 & 4,03 & 18 & 1,38 & 2 & 0,33 & 2 & 0,40 & 6,15 \\
\hline TJF & 107 & 0 & 0,00 & 86 & 2,97 & 4 & 0,31 & 16 & 2,67 & 1 & 0,20 & 6,14 \\
\hline NTJ & 33 & 0 & 0,00 & 1 & 0,03 & 1 & 0,08 & 31 & 5,17 & 0 & 0,00 & 5,28 \\
\hline JBE & 26 & 0 & 0,00 & 1 & 0,03 & 0 & 0,00 & 4 & 0,67 & 21 & 4,20 & 4,90 \\
\hline JMAR & 64 & 0 & 0,00 & 6 & 0,21 & 56 & 4,31 & 1 & 0,17 & 1 & 0,20 & 4,88 \\
\hline JATA & 27 & 0 & 0,00 & 0 & 0,00 & 0 & 0,00 & 27 & 4,50 & 0 & 0,00 & 4,50 \\
\hline AER & 46 & 0 & 0,00 & 26 & 0,90 & 7 & 0,54 & 7 & 1,17 & 6 & 1,20 & 3,80 \\
\hline JFE & 76 & 0 & 0,00 & 70 & 2,41 & 2 & 0,15 & 4 & 0,67 & 0 & 0,00 & 3,23 \\
\hline $\mathrm{AH}$ & 56 & 0 & 0,00 & 49 & 1,69 & 3 & 0,23 & 2 & 0,33 & 2 & 0,40 & 2,65 \\
\hline CAR & 47 & 0 & 0,00 & 37 & 1,28 & 5 & 0,38 & 5 & 0,83 & 0 & 0,00 & 2,49 \\
\hline JPE & 28 & 0 & 0,00 & 17 & 0,59 & 3 & 0,23 & 1 & 0,17 & 7 & 1,40 & 2,38 \\
\hline
\end{tabular}


Os periódicos de maior impacto na pesquisa contábil brasileira e norte-americana: uma análise comparativa baseada nas citações em teses de doutorado
Octavio Ribeiro de Mendonça Neto; Ricardo Lopes Cardoso; José Carlos Tiomatsu Oyadomari

\begin{tabular}{|c|c|c|c|c|c|c|c|c|c|c|c|c|}
\hline TJPE & 16 & 0 & 0,00 & 0 & 0,00 & 5 & 0,38 & 10 & 1,67 & 1 & 0,20 & 2,25 \\
\hline ITPF & 13 & 0 & 0,00 & 0 & 0,00 & 3 & 0,23 & 10 & 1,67 & 0 & 0,00 & 1,90 \\
\hline TAMJ & 21 & 0 & 0,00 & 8 & 0,28 & 8 & 0,62 & 0 & 0,00 & 5 & 1,00 & 1,89 \\
\hline JEB & 8 & 0 & 0,00 & 0 & 0,00 & 0 & 0,00 & 0 & 0,00 & 8 & 1,60 & 1,60 \\
\hline QJE & 22 & 0 & 0,00 & 9 & 0,31 & 11 & 0,85 & 0 & 0,00 & 2 & 0,40 & 1,56 \\
\hline JAPP & 23 & 0 & 0,00 & 17 & 0,59 & 1 & 0,08 & 5 & 0,83 & 0 & 0,00 & 1,50 \\
\hline SMJ & 16 & 0 & 0,00 & 1 & 0,03 & 13 & 1,00 & 2 & 0,33 & 0 & 0,00 & 1,37 \\
\hline AAA & 18 & 0 & 0,00 & 7 & 0,24 & 9 & 0,69 & 0 & 0,00 & 2 & 0,40 & 1,33 \\
\hline TJA & 14 & 0 & 0,00 & 7 & 0,24 & 3 & 0,23 & 2 & 0,33 & 2 & 0,40 & 1,21 \\
\hline FS & 8 & 0 & 0,00 & 1 & 0,03 & 0 & 0,00 & 7 & 1,17 & 0 & 0,00 & 1,20 \\
\hline MA & 13 & 0 & 0,00 & 1 & 0,03 & 11 & 0,85 & 0 & 0,00 & 1 & 0,20 & 1,08 \\
\hline JIFMA & 25 & 0 & 0,00 & 23 & 0,79 & 1 & 0,08 & 0 & 0,00 & 1 & 0,20 & 1,07 \\
\hline HBR & 13 & 0 & 0,00 & 2 & 0,07 & 10 & 0,77 & 0 & 0,00 & 1 & 0,20 & 1,04 \\
\hline ITJ & 6 & 0 & 0,00 & 0 & 0,00 & 0 & 0,00 & 6 & 1,00 & 0 & 0,00 & 1,00 \\
\hline IAE & 5 & 0 & 0,00 & 0 & 0,00 & 0 & 0,00 & 0 & 0,00 & 5 & 1,00 & 1,00 \\
\hline JAL & 9 & 0 & 0,00 & 0 & 0,00 & 6 & 0,46 & 3 & 0,50 & 0 & 0,00 & 0,96 \\
\hline Outros & 942 & 8 & & 427 & & 290 & & 13 & & 115 & & \\
\hline Total & 2310 & 8 & & 1250 & & 587 & & 289 & & 176 & & \\
\hline$\%$ & & 0,3 & & 54,1 & & 25,4 & & 12,5 & & 7,7 & & \\
\hline
\end{tabular}

Fonte: Dados da pesquisa.

Conforme se pode observar na TAB. 5, a área de contabilidade financeira responde por 54, $1 \%$ das citações, seguida da área de contabilidade gerencial, com 25, 4\%. No caso de Chan, Chan, Seow e Tam (2009), os resultados indicaram de 59,8\% para a área de contabilidade financeira, 14,1\% para área de auditoria e 13, $1 \%$ para área de contabilidade gerencial. Interessante notar que, no caso brasileiro, a contabilidade tributária é responsável por um percentual significativo das citações $(12,5 \%)$, enquanto que, no caso norte americano, este número é de apenas 3,9\%. Essa diferença expressiva pode estar ligada a forte influência do fisco na normatização da contabilidade brasileira. Outro aspecto a ser salientado no caso brasileiro, é o número reduzido de teses na área de auditoria e, consequentemente, a pequena participação das citações a ela relacionadas $(0,3 \%)$.

Baseado na métrica proposta por Chan et al. (2009), os cinco periódicos mais citados, no caso brasileiro, foram: Journal of Accounting and Public Policy (JAPP), The Accounting Review (TAR), Accounting, Organizations and Society (AOS), Journal of Accounting Research (JAR) e The Journal of Finance (TJF). 
Uma comparação entre esses resultados e os obtidos por Chan, Chan, Seow e Tam (2009) é apresentada na TAB. 6:

Tabela 6 - Análise comparativa de citações por periódico e por área de especialização

\begin{tabular}{cllll}
\hline & Estudo Atual & & Chan et al. (2009) \\
Número de Teses & 55 & 247 & \\
Citações por tese & 42 & 82 & \\
& & & 59,8 \\
Área de & Financeira & 54,1 & Financeira & 14,1
\end{tabular}

Journal of Accounting and The Accounting Review

Public Policy

Ranking de The Accounting Review Journal of Accounting Research

Periódicos Accounting Organizations Accounting Organizations and Society

and Society

Journal of Accounting Journal of Accounting and Economics

Research

The Journal of Finance

Auditing: A Journal of Practice and Theory

Fonte: Dados da pesquisa.

Estes dados revelam, novamente, uma coincidência em ambos os rankings de três periódicos The Accounting Review (TAR), Accounting, Organizations and Society (AOS), Journal of Accounting Research (JAR), embora não na mesma ordem. No caso norte americano, cabe salientar, ainda, a presença do Auditing: A Journal of Practice and Theory (AUDIT), o que pode ser explicado pelo número expressivo de teses na área de auditoria, o que não acontece para o Brasil.

\subsection{Análise comparativa do ranking de periódicos por método de pesquisa}

O QUADRO 2 apresenta um ranking de periódicos para cada método de pesquisa, baseado no número de citações em teses brasileiras. Neste quadro, esses rankings são comparados com aqueles encontrados por Chan et al. (2009). Deixou-se de considerar a metodologia experimental, em virtude de, no caso brasileiro, ela apenas ter sido utilizada em uma única tese. 
Quadro 2 - Ranking de periódicos por método de pesquisa

\begin{tabular}{|c|c|c|}
\hline Ranking & Estudo Atual & Chan et al. (2009) \\
\hline & Documental & Documental \\
\hline 1 & Journal of Accounting and Public Policy & Journal of Accounting and Economics \\
\hline 2 & The Accounting Review & Journal of Accounting Research \\
\hline 3 & Journal of Accounting Research & The Accounting Review \\
\hline 4 & The Journal of Finance & Contemporary Accounting Research \\
\hline 5 & Journal of Financial Economics & Accounting Horizons \\
\hline & Modelagem & Modelagem \\
\hline 1 & The Journal of Finance & Journal of Accounting Research \\
\hline 2 & $\begin{array}{l}\text { Journal of International Financial } \\
\text { Management and Accounting }\end{array}$ & The Accounting Review \\
\hline 3 & American Economic Review & Journal of Accounting and Economics \\
\hline 4 & Journal of Finance and Quantitative Analysis & Contemporary Accounting Research \\
\hline 5 & The Accounting Review & Review of Accounting Studies \\
\hline & Levantamento & Levantamento \\
\hline 1 & Accounting, Organizations and Society & Accounting, Organizations and Society \\
\hline 2 & Journal of Management Accounting Research & The Accounting Review \\
\hline 3 & The Accounting Review & Journal of Accounting Research \\
\hline 4 & Journal of Accounting Research & Management Accounting \\
\hline 5 & Journal of Accounting and Public Policy & Journal of Management Accounting Research \\
\hline
\end{tabular}

Fonte: Dados da pesquisa.

Esses resultados confirmam os achados de Chan et al. (2009), que sugerem rankings diferentes para métodos de pesquisas diferentes.

A análise comparativa dos rankings revela, para o caso da pesquisa documental, uma coincidência de dois periódicos, The Accounting Review (TAR) e Journal of Accounting Research (JAR). Cabe observar, ainda, com relação à pesquisa documental, que o Accounting Horizons $(A H)$ e 0 Contemporary Accounting Research (CAR), que figuram entre os cinco primeiros no ranking de Chan et al. (2009), aparecem no presente estudo, na sexta e sétimas posições, respectivamente, enquanto que o Journal of Accounting and Economics (JAE), primeiro do ranking norte-americano, não figura, neste estudo, entre os 30 mais citados. 
Com relação às teses que utilizaram como método de pesquisa a modelagem, o único periódico que aparece nos dois rankings é o The Accounting Review (TAR).

Já no caso das pesquisas que utilizaram o levantamento, observa-se que quatro periódicos são comuns aos dois rankings, embora não na mesma ordem. São eles: Accounting, Organizations and Society (AOS), Journal of Management Accounting Research (JMAR), The Accounting Review (TAR) e Journal of Accounting Research (JAR).

\subsection{Análise comparativa do ranking de periódicos por área de especialização}

O QUADRO 3 apresenta um ranking de periódicos para cada área de especialização da contabilidade, baseado no número de citações em teses brasileiras.

Neste quadro, esses rankings também são comparados com aqueles encontrados por Chan et al. (2009). Nesse caso, deixou-se de considerar área de auditoria em virtude do reduzido número de teses nessa área no Brasil (três teses).

Quadro 3 - Ranking dos periódicos por área de especialização

\begin{tabular}{|c|c|c|}
\hline Ranking & Estudo Atual & Chan et al. (2009) \\
\hline & Financeira & Financeira \\
\hline 1 & Journal of Accounting and Public Policy & Journal of Accounting Research \\
\hline 2 & The Accounting Review & Journal of Accounting and Economics \\
\hline 3 & Journal of Accounting Research & The Accounting Review \\
\hline 4 & The Journal of Finance & Contemporary Accounting Research \\
\hline \multirow[t]{2}{*}{5} & Journal of Financial Economics & Accounting Horizons \\
\hline & Gerencial & Gerencial \\
\hline 1 & Accounting, Organizations and Society & The Accounting Review \\
\hline 2 & Journal of Management Accounting Research & Accounting, Organizations and Society \\
\hline 3 & The Accounting Review & Journal of Accounting Research \\
\hline 4 & Journal of Accounting Research & Journal of Management Accounting Research \\
\hline \multirow[t]{2}{*}{5} & Journal of Accounting and Public Policy & Journal of Accounting and Economics \\
\hline & Tributária & Tributária \\
\hline 1 & National Tax Journal & Journal of the American Taxation Association \\
\hline 2 & Journal of the American Taxation Association & National Tax Journal \\
\hline 3 & The Accounting Review & The Accounting Review \\
\hline 4 & The Journal of Finance & Journal of Accounting Research \\
\hline 5 & Journal of Accounting and Public Policy & Journal of Accounting and Economics \\
\hline
\end{tabular}

Fonte: Dados da pesquisa. 
Também, nesse caso, os resultados obtidos confirmam os achados de Chan, Chan, Seow e Tam (2009), que sugerem rankings diferenciados para cada área específica da contabilidade. A análise comparativa dos rankings, apresentada no QUADRO 3, revela, para o caso da área de contabilidade financeira, que dois periódicos, The Accounting Review (TAR) e Journal of Accounting Research (JAR) são comuns a ambos. Cabe observar, ainda, que da mesma forma que no ranking relacionado à pesquisa documental, apresentado no item 4.5, o Accounting Horizons $(\mathrm{AH})$, o Contemporary Accounting Research (CAR), que figuram entre os cinco primeiros no ranking de Chan, Chan, Seow e Tam (2009), também aparecem no presente estudo na sexta e sétimas posições respectivamente, enquanto que o Journal of Accounting and Economics JAE, não figura, nesse estudo, entre os 30 mais citados.

Já no caso da contabilidade gerencial, os periódicos Accounting, Organizations and Society (AOS), Journal of Management Accounting Research (JMAR), The Accounting Review (TAR) e Journal of Accounting Research (JAR), aparecem entre os cinco primeiros em ambos os rankings, o mesmo acontecendo com National Tax Journal (NTA), Journal of the American Taxation Association (JATA) e The Accounting Review $(T A R)$, para o caso da contabilidade tributária.

\section{Considerações finais}

A importância da publicação em periódicos científicos, como uma forma de avaliar a produção acadêmica, tem motivado vários pesquisadores a se preocuparem em desenvolver modelos de rankings de qualidade desses periódicos (BEATTIE; GOODACRE, 2006). Essa importância também é salientada por vários autores (READ; RAMA; RAGHUNANDAN, 1998; ARLINGHAUS, 2002; MATHERLLY; SHORTRIDGE, 2009), que observam que a qualidade dos periódicos nos quais os pesquisadores publicam, constitui um aspecto importante na avaliação da sua produtividade acadêmica e, consequentemente, influi nas decisões relacionadas à evolução na carreira, empregabilidade, salário, dentre outros.

Além disso, essa produtividade constitui um aspecto importante no processo de credenciamento, avaliação e reputação dos programas de pós-graduação em que esses acadêmicos atuam (SINNIG; DYKXHOORN, 2001), avaliação essa que, no caso brasileiro, é conduzido pela Coordenação de Aperfeiçoamento de Pessoal de Nível Superior (CAPES), à semelhança do Association of Advance Collegiate Schools of Business $(A A C S B)$, para o caso norte-americano.

O presente estudo teve por objetivo apresentar um ranking dos periódicos de língua inglesa com maior influência sobre a formação do pensamento contábil no Brasil, baseado nas citações em teses de 
doutorado. Utilizou, deliberadamente, a mesma metodologia desenvolvida por Chan et al. (2009) e apresentou uma análise comparativa dos achados de ambas as pesquisas.

Os resultados obtidos por este estudo confirmam aqueles encontrados por Chan et al. (2009) e sugerem a adoção de rankings diferenciados, de acordo com as áreas de específicas da contabilidade ou com os métodos de pesquisa utilizados.

Esta sugestão reveste-se de uma importância significativa, já que o ranking de periódicos elaborado pela Coordenação de Aperfeiçoamento de Pessoal de Nível Superior (CAPES), órgão oficial que credencia e avalia os programas de pós-graduação e consequentemente a produtividade dos acadêmicos desses programas, é único, não considerando nem a área específica da contabilidade em que se enquadra a linha de pesquisa desse acadêmico e nem o método de pesquisa utilizado pelo mesmo.

Com relação à análise comparativa entre os resultados obtidos nesse estudo e aqueles encontrados por Chan et al. (2009), cabem as considerações abaixo.

O método de pesquisa mais utilizado, em ambos os casos, é a documental. Sob esse aspecto, os resultados sugerem um baixo grau de alinhamento dessa modalidade de pesquisa entre os dois países, já que dentre os cinco periódicos mais citados pelos pesquisadores brasileiros, apenas dois figuram entre os cinco mais citados pelos pesquisadores norte-americanos. Este grau de alinhamento é bem mais elevado para o caso da pesquisa por levantamento, em que quatro dos cinco periódicos mais citados, no Brasil, figuram entre os cinco primeiros do ranking de Chan et al. (2009) (ver QUADRO 2).

Em termos de área de especialização, a contabilidade financeira é a mais escolhida nos dois países, e responsável por mais da metade das teses defendidas em ambos os casos. Os resultados obtidos, nesse caso, também sugerem um baixo grau de alinhamento das pesquisas, já que, novamente, apenas dois periódicos dentre os cinco mais citados por pesquisadores brasileiros figuram entre os cinco mais citados por pesquisadores norte-americanos. No caso da área de contabilidade gerencial, todavia, o grau de alinhamento já é bem mais elevado, uma vez que quatro periódicos dentre os cinco primeiros do ranking brasileiro figuram entre os cinco primeiros do ranking norte-americano. Já para a contabilidade tributária, os resultados sugerem um grau de alinhamento um pouco menor com três periódicos dentre os cinco mais citados no Brasil, figurando entre os cinco mais citados por norte-americanos (ver QUADRO 3). 
A coerência desses resultados pode ser constada, cruzando-se o grau de alinhamento das áreas de especialização com o grau de alinhamento da metodologia utilizada. No caso da contabilidade financeira, na qual o grau de alinhamento é baixo, o método de pesquisa mais utilizado é o da pesquisa documental, em que o grau de alinhamento também é baixo. O contrário ocorre com a contabilidade gerencial, na qual o método de pesquisa mais utilizado é o do levantamento.

A principal limitação deste estudo está relacionada ao fato de que todas as 55 teses analisadas foram desenvolvidas por doutorandos de uma única universidade (USP). Isso, conforme já observado, decorre do fato de que durante o período em análise, a USO era a única universidade brasileira a oferecer curso de doutorado na área de ciências contábeis. Esse fato pode ter introduzido algum viés, quando da análise comparativa com os resultados de Chan et al. (2009), já que esses utilizaram teses de 84 universidades.

Para futuras pesquisas, sugere-se uma análise crítica sobre as consequências dessa influência dos periódicos de língua inglesa sobre a contabilidade brasileira, considerando principalmente os aspectos sociológicos envolvidos. Um estudo dessa natureza permitirá que se reflita se a pesquisa contábil e a formação dos pesquisadores, que vem sendo realizada no Brasil, atende aos interesses da profissão e da sociedade brasileira, de uma forma mais geral.

\section{Referências}

ARLINGHAUS, B. P. The environment for professional interaction and relevant practical experience in AACSB - accredited accounting programs. Journal of Education for Business, v. 78, n.1, p. 38-45, 2002.

BALLAS, A.; THEOHARAKIS, V. Exploring diversity in accounting through faculty journal perceptions. Contemporary Accounting Research, v. 20, n. 4, p. 619-644, 2003.

BEAN, D. F.; BERNARDI, R. A. Estimating the ratings of journals omitted in prior quality ratings. Advances in accounting education, v.7, p. 109$127,2005$.

BEATTIE, V.; GOODACRE, A. A new method for ranking academic journals in accounting and finance. Accounting and Business Research, v. 36, n. 2, p. $65-91,2006$.

BONNER, S. E. et al. (2006), The most influential journals in academic accounting. Accounting, Organizations and Society, v. 31, n. 7, p. 663$685,2006$.

BROWN, L. D.; HUEFNER, R. J. The familiarity with and perceived quality of accounting journals: views of senior accounting faculty in leading U.S. 
MBA programs. Contemporary Accounting Research, v. 11, n.1, p. 223250, 1994.

BROWN, L. D. Ranking journals using Social Science Research Network downloads. Review of Quantitative Finance and Accounting, v. 20, n. 3, p. 291-307, 2003.

CHAN, K. C. et al. Ranking accounting journals using dissertation citation analysis: a research note. Accounting, Organizations and Society, v. 34, n. $6 / 7$, p. 875-885, 2009.

CHAN, K. C.; LIANO, K. A threshold citation analysis of influential articles, journals, institutions, and researchers in accounting apud CHAN, K. C. et al. Ranking accounting journals using dissertation citation analysis: a research note. Accounting, Organizations and Society, v. 34, n. 6/7, p. 875-885, 2009.

GARFIELD, E. Citation indexing: its theory and application in science, technology and humanities. New York: John Wiley \& Sons Inc., 1979.

HERRON, T. L.; HALL, T. W. Faculty perceptions if journals: quality and publishing feasibility. Journal of Accounting Education, v. 22, n. 3, p. 175210, 2004.

JOHNSON, P. M.; RECKERS, P. M. J.; SOLOMON, L. Evolving research benchmarks. Advances in Accounting, v. 19, p. 235-243, 2002.

JOLLY, S. A.; SCHOEDER, R. G.; SPEAR, R. K. An empirical investigation of the relationship between journal quality ratings and promotion and tenure decisions. Accounting Educators Journal, v. 7, n. 1, p. 47-68, 1995.

LOWENSOHN, S.; SAMELSON, D. P. An examination of faculty perceptions of academic journal quality within five specialized areas of accounting research. Issues in Accounting Education, v. 21, n. 3, p. 219- 239, 2006.

LUKKA, K.; KASANEN, E. Is accounting a global or a local discipline? Evidence from major research journals. Accounting Organizations and Society, v. 21, n. 7/8, p. 755-773, 1996.

MATHERLY, M.; SHORTRIDGE, R. T. A pragmatic model to estimate journal quality in accounting. Journal of Accounting Education, v. 27, n. 1, p. 14-29, 2009.

READ, W. J.; RAMA, D. V.; RAGHUNANDAN, K. Are publications requirements for accounting faculty promotions still increasing? Issues in Accounting Education, v.13, n.2, p. 327-339, 1998.

REINSTEIN, A.; CALDERON, T. G. Examining accounting departments rankings of the quality of accounting journals. Critical Perspectives on Accounting, v.17, n. 4, p. 457-490, 2006.

SINNING, K. E.; DYICXHOORN, H. J. J. Process implemented for AACSB, accounting accreditation and the degree of faculty involvement. Issues in Accounting Education, v.16, n.2, p. 181-204, 2001. 
Os periódicos de maior impacto na pesquisa contábil brasileira e norte-americana: uma análise comparativa baseada nas citações em teses de doutorado
Octavio Ribeiro de Mendonça Neto; Ricardo Lopes Cardoso; José Carlos Tiomatsu Oyadomari

SMITH, L. M. Relative contributions of professional journals to the field of accounting. Accounting Educators Journal, v. 6, n. 1, p. 1-31, 1994. 